Article

\title{
Association of Smoking Prevalence and Alcohol Use with Risk of Lung Cancer: Ecological Evidence
}

\author{
Yuan Jiang ${ }^{1, *}$, Chi Zhang ${ }^{2}$, Fengyu Zhang ${ }^{3, *}$ \\ 1 Tobacco Control Office, the Center for Disease Control and Prevention, Xicheng \\ District, Beijing, China \\ 2 The University of Maryland, College Park, MD, USA \\ 3 The Global Clinical and Translational Research Institute, Bethesda, MD, USA \\ * Correspondence: Fengyu Zhang, Email:zhangfy@gcatresearch.org; Yuan Jiang, \\ Email: jiangyuan88@vip.sina.com.
}

\section{ABSTRACT}

Background: Smoking and alcohol use are two common behaviors that affect human health. However, few studies have examined their association with risk of lung cancer with ecological data.

Methods: The dependent variable was the estimate of world agestandardized incidence of lung cancer in 2018. Independent variables included the smoking prevalence by gender in 2010 and 2000, which considered potential latency of lung cancer, the rate of alcohol use by gender in 2010, and the gross domestic product (GDP) per capita in 2010 and 2000. A linear regression model was used for statistical data analysis.

Results: Both simple and multiple linear regression analysis showed that the prevalence of women's smoking affected the incidence of lung cancer overall ( $t=4.96 ; P<0.0001)$, in men $(t=4.56 ; P<0.0001)$ and in women $(t=3.22 ; P<0.0001)$ even when socioeconomic development measured by the GDP per capita was adjusted. These associations were consistent in the overall $(t=4.59 ; P<0.0001)$ and men $(t=3.37 ; P=0.001)$, but stronger in women ( $t=4.67 ; P<0.0001)$ when the estimates of smoking prevalence in 2000 were used; and the GDP per capita was significantly associated

\section{G Open Access}

Received: 23 October 2018 Accepted: 04 January 2019 Published: 19 March 2019

Copyright (c) 2019 by the author(s). Licensee Hapres, London, United Kingdom. This is an open access article distributed under the terms and conditions of Creative Commons Attribution 4.0 International License. with the incidence of lung cancer as well. Interestingly, the ratio of smoking prevalence between men and women in 2000 explained about $10 \%\left(P=0.0006 ; R^{2}=0.097\right)$ of the variation in the ratio of lung cancer incidence between men and women even after adjusting for socioeconomic development. However, we did not find an association of alcohol use with the incidence of lung cancer at an aggregated level.

Conclusion: At an aggregated level, the women's smoking prevalence is associated with the incidence of lung cancer and gender difference in smoking is associated with the ratio of lung cancer incidence between men and women, providing strong evidence for the relationship of smoking and lung cancer. 
KEYWORDS: smoking prevalence; alcohol use; the incidence of lung cancer; ecological analysis; sex difference

\section{INTRODUCTION}

Smoking and alcohol use along with physical inactivity and diet are common behaviors that affect human health [1]. Smoking in forms of cigarette or tobacco smoking may begin in adolescence or early childhood mostly due to perceived pressure or desire to respond to social peer pressure. In general, more men than women smoke. Since the 1960s, the rate of smoking has peaked or declined in the more developed world, but it continued to increase in the less developed world according to the WHO in 2015. Individual personality factors, cognitive factors, and coping resources such as family and social support may play a key role in determining the initiation and continuation of smoking [2]. Alcohol consumption is another common human behavior, and according to the National Survey on Drug Use and Health (NSDUH) in the United States, more than half of individuals aged at 26 years or older reported that they were current alcohol users [3]. While smoking is mostly believed to harm health, the effect of alcohol use varies from person to person, depending on a variety of factors, including how much and how often one drinks, alcohol metabolism, and body size.

Lung cancer is a malignant tumor formed by the uncontrolled growth of abnormal cells that start in one or both lungs, usually in the cells that line the airway passages. The mortality of lung cancer has a gender difference. While mortality of lung cancer has started to decline in males in all continents except Africa, likely due to smoking cessation and improved therapeutics, but it continues to rise in females in most of the continents [4]. The pattern of lung cancer incidence is entirely different across populations, with a higher incidence in the more developed countries in Europe and North America, but also in the rapidly developing such as China. In the United States, apart from the predominantly gender-specific breast and prostate cancers, lung cancer has the highest incidence, and this is seen consistently across different major ethnic groups. According to the International Agency for Research on Cancer (IARC) in 2016 [5], the world age-standardized rate of incidence of lung cancer was 44.2 per 100,000 individuals and was by far the leading cause of cancer death among both men and women in the US. Twenty-five percent of cancer deaths are from lung cancer, in which each year, more people die of lung cancer than the other three most prevalent cancers including breast, prostate cancer, and colon cancer combined. However, for China, lung cancer ranked first by incidence in both men and women. Its world age-standardized rate of the incidence of lung cancer in 2016 was 52.8 per 100,000 populations, exceeding that of the United States. While China has a different pattern of cancer from the Western world, lung cancer in China accounts for about 22 percent of 
global new cancer cases, and about 27 percent of global cancer deaths occur in China.

Previous studies have shown strong evidence for cigarette smoking as a risk factor for lung cancer but mostly at an individual level. The association of smoking and lung cancer was discovered by two landmark studies in the 1950s [6,7]. The potential mechanism considered was that cigarette smoke contains known carcinogens and high levels of reactive oxygen species, which lead to an impairment of epithelial and endothelial cell function as well as inflammation [8]. However, the association of alcohol drinking with lung cancer is more complicated to determine, because it depends on how much and what kind of alcohol beverage people drink. For example, drinking red wine has been considered as a disease prevention practice, but drinking alcoholic beverages appear to increase the risk of breast cancer in women-the general view that alcohol use is associated with lung cancer even after adjusting for smoking [9]. However, most studies were limited to factors measured for individuals with few considering data at the ecological level. Ecological data are important for lung cancer because people who live in different geographic locations may be suffering from varying degrees of air pollutions or deficiency in trace elements in their diets that have been associated with risk of cancers [10]. Data at an ecological level may capture some unobserved variable or unobservable heterogeneity such as air quality, culture, a social system that may affect both risks of lung cancer and human behaviors among individuals.

In this study, we aimed to examine the association of smoking prevalence and alcohol use with the incidence of lung cancer to determine how two important health behaviors affect the development of lung cancer at the ecological level. We first examined the association of smoking prevalence and rate of alcohol use by gender with the overall and gender-specific incidence for lung cancer, and then a multiple regression analysis was performed to determine the significant factors associated with lung cancer by gender while adjusting for the level of socioeconomic development. Finally, we examined the association of gender ratio of the incidence of lung cancer with the rate of smoking and alcohol use between men and women.

\section{METHODS}

\section{Variables and Data Source}

The dependent variable was the incidence of lung cancer overall and by gender. The data was based on the latest release of the GLOB-OCAN database (September 2018) that includes estimates of the incidence of lung cancer in 185 countries or region around the world and by gender. It is available at the IARC Global Cancer Observatory website (http://gco.iarc.fr/). 
The independent variables were the prevalence of smoking and alcohol drinking by gender. Considering data availability and that lung cancer may have a period of latency, we selected the prevalence of smoking in 2000 and 2010 and the percentage of alcohol drinking by adults in 2010. According to the data source used, smoking was defined as a percentage of women and men aged 15 years and older who smoke any form of tobacco, including cigarettes, cigars, pipes or any other smoked tobacco products on a daily, nondaily or occasional basis. The data was published by World Bank-World Development Indicators, which is available at the World Health Organization (WHO), Global Health Observatory Data Repository [11]. The proportion of adults who drank alcohol last year (2010) measured alcohol use, and non-alcohol users defined as individuals at age 15 years or older who have not consumed any alcohol during the past 12 months. All these data were downloaded from https://ourworldin-data.org/.

Socioeconomic development may be factors that affect smoking and alcohol use behaviors or other factors that may contribute to lung cancer incidence and mortality. In fact, except for China, lung cancer is more prevalent in the more developed countries, suggesting that lung cancer may be associated with socioeconomic development. We only used these variables as covariates for assessing smoking and alcohol use with lung cancer. Socioeconomic development was measured by the gross domestic product (GDP) per capita (in US dollars) and human development index (HDI), derived by the World Bank; and data in 2000 and 2010 was downloaded from the website, https://data.worldbank.org/indicator/. Both factors are highly correlated at a country level. All data were downloaded on September 25, 2018.

\section{Statistical Analysis}

Simple linear regression analysis was first performed for determining an association of the incidence of lung cancer with the prevalence of smoking and alcohol use overall and by gender, and then multiple linear regression analysis of lung cancer incidence was performed with the prevalence of smoking and alcohol use by gender while adjusting for socioeconomic status. We also analyzed the ratio of lung cancer incidence with smoking and alcohol use to examine how these gender differences in the incidence of lung cancer can be explained by the difference in smoking and alcohol use between men and women. The coefficient of determination ( $R$-square, $R^{2}$ ) was used to measure the contribution of an individual variable(s) to the ecological variation in the incidence of lung cancer.

\section{RESULTS}

Table 1 presents the summary statistics for lung cancer, smoking prevalence, and alcohol use. In general, the ratio of the median incidence of lung cancer between men and women was about threefold. In both 
genders, the countries with lung cancer incidence in the top quarter ( $\mathrm{W} \geq 25 \%$ ) were mostly in Europe, North America, and China. The median smoking rate was $34.8 \%$ in men, and $9.6 \%$ in women in 2010 , which was an almost 4-fold difference, but the prevalence of smoking in both men and women were decreased compared to the year 2000. The median rate of alcohol use was $55.8 \%$ in men and $32.6 \%$ in women, about a 1.6-fold difference.

Table 1. Descriptive statistics of the incidence of lung cancer, smoking prevalence and alcohol drinking by gender.

\begin{tabular}{lrrrrrrr}
\hline Variable & $\boldsymbol{N}$ & Mean & Median & Min & 25th Pctl & 75th Pctl & Max \\
\hline Year 2018 & & & & & & & \\
The incidence of lung cancer & 169 & 15.55 & 12.4 & 0.5 & 5.1 & 25.3 & 56.7 \\
Incidence of men & 169 & 22.78 & 17.4 & 0.8 & 6.4 & 35.7 & 77.4 \\
Incidence in women & 169 & 9.63 & 6.0 & 0.2 & 2.9 & 13.5 & 41.4 \\
Incidence ratio of men to women & 169 & 2.89 & 2.32 & 0.8 & 1.6 & 3.3 & 10.6 \\
Year 2010 & & & & & & & \\
Smoking in men (\%) & 117 & 35.74 & 34.8 & 9.0 & 26.5 & 44.8 & 82.0 \\
Smoking in women (\%) & 118 & 12.53 & 9.6 & 0.5 & 3.2 & 21.3 & 40.2 \\
The smoking ratio of men to women & 117 & 9.64 & 3.81 & 0.56 & 1.6 & 11.0 & 99.8 \\
Alcohol drinking in men (\%) & 168 & 50.39 & 55.8 & 3.0 & 25.8 & 71.2 & 96.5 \\
Alcohol drinking in women (\%) & 168 & 34.07 & 32.6 & 0.2 & 10.1 & 51.2 & 93.3 \\
The ratio of men to women & 168 & 2.05 & 1.64 & 1.02 & 1.3 & 2.3 & 17.5 \\
GDP per capita (US\$) & 163 & 13,150 & 4,683 & 231 & 1,326 & 15,959 & 104,965 \\
Human Development Index (0-1) & 164 & 0.684 & 0.714 & 0.351 & 0.544 & 0.808 & 0.942 \\
Year 2000 & & & & & & & \\
Smoking in men (\%) & 117 & 38.82 & 38.4 & 9 & 27.5 & 51.2 & 71.8 \\
Smoking in women (\%) & 118 & 15.76 & 13.1 & 0.6 & 4.8 & 26.1 & 43.6 \\
The smoking ratio of men to women & 117 & 6.72 & 3.22 & 0.29 & 1.44 & 8.18 & 97.7 \\
GDP per capita (US\$) & 117 & 12787 & 3955 & 197 & 1287 & 18263 & 93463 \\
Human Development Index (0-1) & 115 & 0.653 & 0.678 & 0.283 & 0.53 & 0.785 & 0.917 \\
\hline
\end{tabular}

Data source: the incidence of lung cancer was in 2018, measured as world age-standardized rate, number of patient per 100,000 populations; smoking and alcohol use data were in 2010. It is believed that the latency of smoking and lung cancer was lagged for about 20 years. Pctl, percentile.

\section{Smoking and Incidence of Lung Cancer}

The prevalence of women's smoking, but not men's smoking was associated with the overall incidence of lung cancer. As shown in Figure 1a, that the men's smoking prevalence was not significantly associated $(P=0.45)$ with the incidence of lung cancer (Figure 1a); whereas the women's smoking was significantly associated $(t=10.91$; $P<0.0001)$ with the incidence of lung cancer and explained more than half (the coefficient of determination, Nagekelke's $R^{2}=0.5147$ ) of the geographic variation in incidence of lung cancer (Figure 1b). 
Gender-specific analyses indicated that men's smoking was marginally associated $(t=1.8 ; P=0.074)$ with the incidence of lung cancer in men and explained $2.75 \%\left(R^{2}=0.0275\right)$ of variation (Figure $1 \mathrm{c}$ ); whereas women's smoking was significantly associated $(t=9.13 ; P<0.0001)$ with the incidence of lung cancer in women and explained $41.83 \%\left(R^{2}=0.4183\right)$ of variation in the incidence (Figure 1d). The cross-analysis showed that men's smoking prevalence was not associated with the incidence of lung cancer in women (Figure 1e), but the women's smoking prevalence was significantly associated $(t=9.6 ; P<0.0001)$ with the incidence of lung cancer in women and explained $44.25 \%\left(R^{2}=0.4425\right)$ of the variation in the risk in women (Figure 1f).
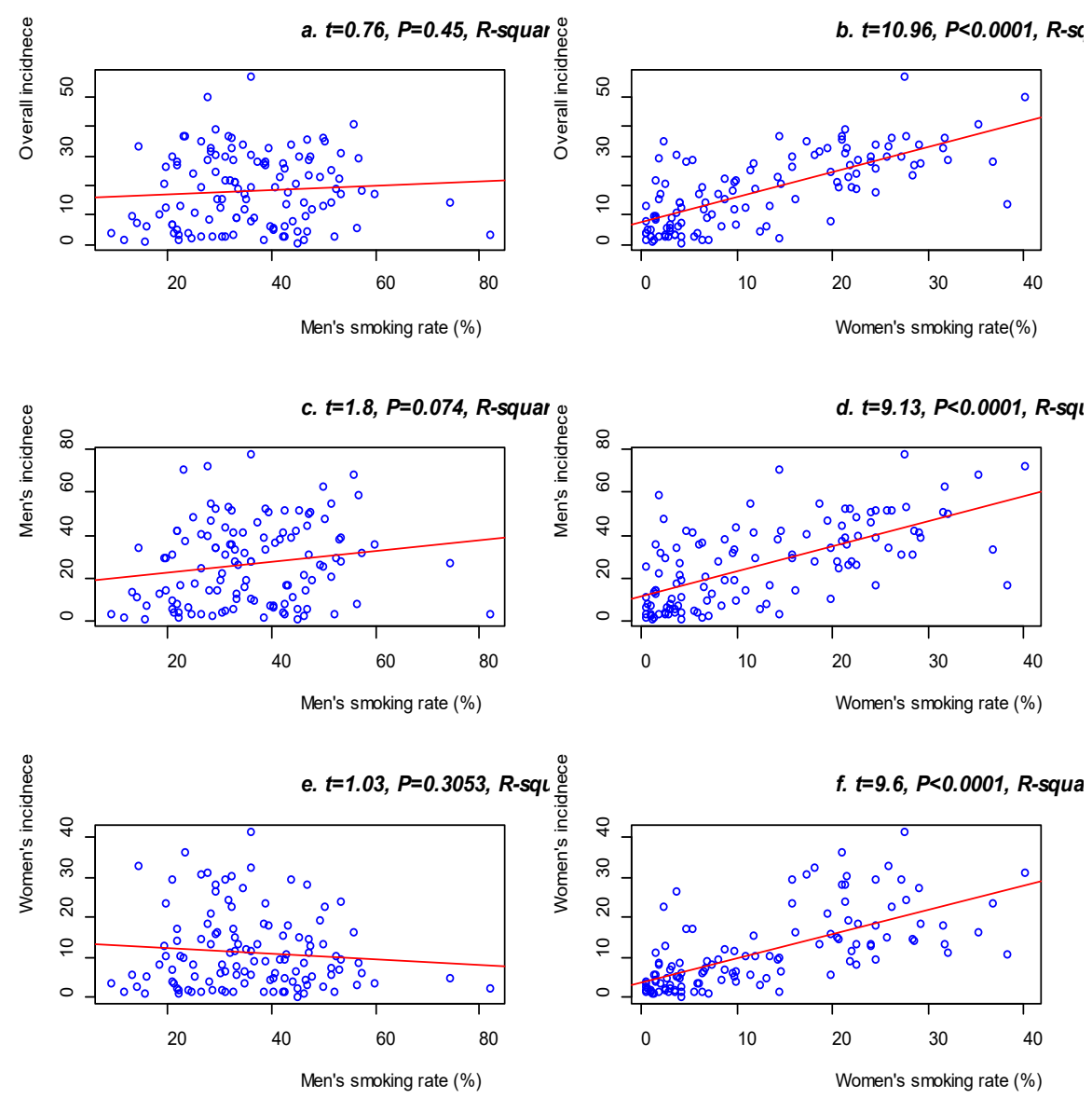

Figure 1. Simple linear regression fitted line on the smoking prevalence and the age-standardized incidence of lung cancer by gender. (a) Fitted results of the overall incidence with the men's smoking; (b) Fitted results of the overall incidence with the women's smoking; (c) Fitted results of the men's incidence with the men's smoking; (d) Fitted results of women's incidence with the women's smoking; (e) Fitted results of the women's incidence with the men's smoking; (f) Fitted results of the women's incidence with women's smoking.

Prevalence of women's smoking was consistently associated with the incidence of lung cancer when multiple regression analysis was performed on both women and men's smoking simultaneously (Table 2). In multiple linear regression analysis, the women's smoking was significantly associated with the incidence of lung cancer overall ( $t=10.91 ; P<0.0001)$, 
in men $(t=8.98 ; P<0.0001)$ and in women $(t=9.77 ; P<0.0001)$. However, men's smoking still showed a marginal association $(t=1.71 ; P=0.090)$ with the incidence in men but even a slight negative association $(t=-2.07$; $P=0.040$ ) with the incidence in women. We noted there was no correlation between women and men's smoking rates. Smoking in men and women together explained about $51.3 \%\left(R^{2}=0.513\right)$ of the variation in the incidence of lung cancer overall, $43.3 \%\left(R^{2}=0.433\right)$ in men and $46.6 \%\left(R^{2}=0.466\right)$ in women. It is interesting to note that when we used the prevalence of smoking in the year 2000, which was about 18 years ahead of the lung cancer incidence data, the association was mostly consistent, with a slightly weaker effect on the incidence of lung cancer overall $\left(R^{2}=0.489 v s .0 .513\right)$ and in men $\left(R^{2}=0.381\right.$ vs. 0.430$)$ but stronger in women $\left(R^{2}=0.511\right.$ vs. 0.460) (Table 2). This association further supports the point that women's smoking prevalence is associated with the incidence of lung cancer in women.

Table 2. Multiple linear regression analysis of the smoking prevalence of men and women with an age-standardized incidence of lung cancer by gender.

\begin{tabular}{|c|c|c|c|c|c|c|c|c|}
\hline \multirow[b]{2}{*}{ Independent variable } & \multicolumn{3}{|c|}{2010} & \multirow[b]{2}{*}{$R^{2}$} & \multicolumn{3}{|c|}{2000} & \multirow[b]{2}{*}{$\boldsymbol{R}^{2}$} \\
\hline & Beta & SE & $\boldsymbol{P}$ & & Beta & SE & $\boldsymbol{P}$ & \\
\hline Overall & & & & 0.513 & & & & 0.489 \\
\hline Men's smoking rate & 0.0199 & 0.0632 & 0.7542 & & 0.0397 & 0.0556 & 0.4773 & \\
\hline Women's smoking rate & 0.8414 & 0.0771 & $<0.0001$ & & 0.6869 & 0.0680 & $<0.0001$ & \\
\hline Men & & & & 0.430 & & & & 0.381 \\
\hline Men's smoking rate & 0.1777 & 0.1039 & 0.0900 & & 0.2143 & 0.0930 & 0.0229 & \\
\hline Women's smoking rate & 1.1382 & 0.1268 & $<0.0001$ & & 0.8506 & 0.1137 & $<0.0001$ & \\
\hline Women & & & & 0.461 & & & & 0.511 \\
\hline Men's smoking rate & -0.1055 & 0.0509 & 0.0404 & & -0.0940 & 0.0416 & 0.0258 & \\
\hline Women's smoking rate & 0.6065 & 0.0621 & $<0.0001$ & & 0.5553 & 0.0509 & $<0.0001$ & \\
\hline
\end{tabular}

Incidence, age-standardized incidence of lung cancer in 2018; Independent variables were smoking rate of men and women in 2000 and 2010; $R^{2}, R$-square, the coefficient of determination.

\section{Alcohol Use and Lung Cancer Incidence}

While alcohol use in both men and women was associated with the incidence of lung cancer, the women's alcohol use had a stronger association than men's alcohol use in simple linear regression analysis. The overall incidence of lung cancer strongly associated with the use of alcohol in men $(t=8.36 ; P<0.0001)$ and women $(t=10.49 ; P<0.0001)$, which explained $29.6 \%\left(R^{2}=0.296\right)$ and $39.9 \%\left(R^{2}=0.399\right)$ of the variation in the incidence of lung cancer (Figure 2a,b). Gender-specific analysis showed that the incidence of lung cancer in men associated with the alcohol use in both men $(t=7.32 ; P<0.0001)$ and women $(t=8.57$; $P<0.0001)$ (Figure 2c,d), which explained about $24.4 \%\left(R^{2}=0.244\right)$ and $30.7 \%\left(R^{2}=0.307\right)$ of the variation in the risk of lung cancer. Similarly, the incidence of lung cancer in women also associated with alcohol use in 
both men $(t=7.32 ; P<0.0001)$ and women $(t=8.57 ; P<0.0001)$ (Figure 2e,f), which explained about $29.9 \%\left(R^{2}=0.299\right)$ and $43.0 \%$ $\left(R^{2}=0.430\right)$ of the variation in the risk of lung cancer. Multiple linear regression analysis of men and women's alcohol use together confirmed that women's alcohol use, but not the men's use of alcohol was associated with the lung cancer incidence overall, in men and women (Data not shown).
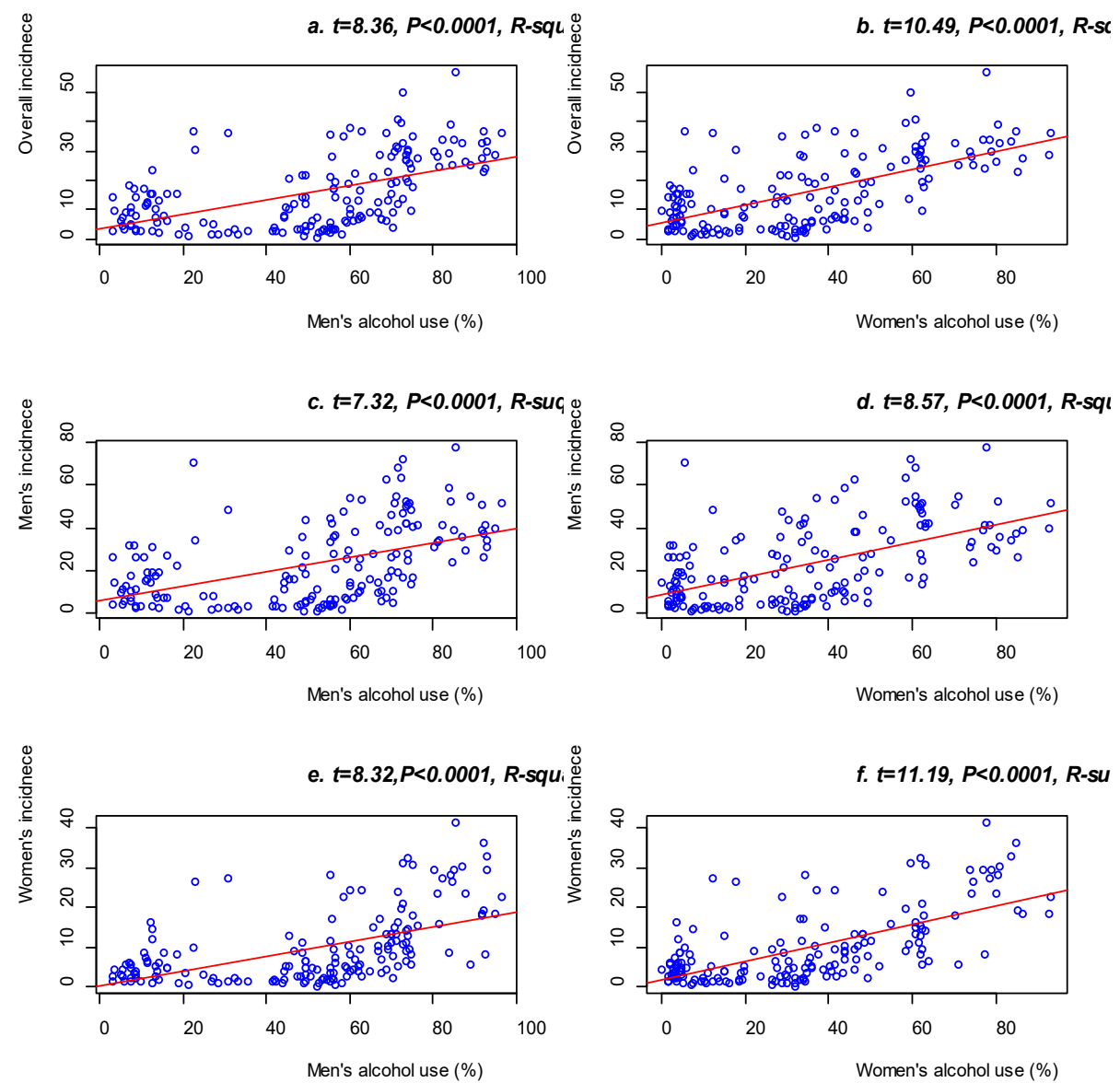

Figure 2. Simple linear regression fitted line on alcohol use in year 2010 and the age-standardized incidence of lung cancer by gender. (a) Fitted results of the overall incidence on men's alcohol use; (b) fitted results of the overall incidence on women's alcohol use; (c) fitted results of men's incidence on men's alcohol use; (d) fitted results of women's incidence on women's alcohol use; (e) fitted results of women's incidence on men's alcohol use; (f) fitted results of women's incidence on women's alcohol use.

\section{Women's Smoking and Alcohol Use with Incidence of Lung Cancer}

Women's smoking prevalence was still consistently associated with the incidence of lung cancer overall and by gender when examining smoking and alcohol use in men and women simultaneously. With the data in the year 2010, only the women's smoking prevalence was associated $(t=5.27 ; P<0.0001)$ with the incidence of lung cancer overall. Gender-specifically, the prevalence of smoking in men $(t=1.99 ; P=0.049)$ and in women $(t=4.86 ; P<0.0001)$ were associated with lung cancer incidence in men. However, the women's smoking prevalence $(t=3.64$; 
$P=0.0004)$ and alcohol using $(t=3.35 ; P=0.0011)$ were significant factors for the incidence of lung cancer in women (Table 3). Women's smoking was associated with the incidence of lung cancer overall, in men and women, but women's alcohol use was only associated with the incidence of lung cancer in women. When the smoking data in 2000 was analyzed, the associations were largely consistent; the prevalence of smoking and alcohol use together explained more variation in the incidence of lung cancer in women from $54.2 \%\left(R^{2}=0.542\right)$ in 2010 to $58.1 \%\left(R^{2}=0.581\right)$, but less variation in men, from $45 \%\left(R^{2}=0.45\right)$ to $42 \%\left(R^{2}=0.42\right)$.

Table 3. Multiple regression analysis of the incidence of lung cancer with smoking and alcohol use by gender based on data in 2010 and $2000 *$.

\begin{tabular}{|c|c|c|c|c|c|c|c|c|c|}
\hline \multirow{2}{*}{ Incidence } & \multirow{2}{*}{$\begin{array}{l}\text { Independent } \\
\text { variable }\end{array}$} & \multicolumn{3}{|c|}{2010} & \multicolumn{5}{|c|}{2000} \\
\hline & & Beta & SE & $\boldsymbol{P}$ & $R^{2}$ & Beta & SE & $\boldsymbol{P}$ & $\boldsymbol{R}^{2}$ \\
\hline \multirow[t]{5}{*}{ Overall } & & & & & 0.551 & & & & 0.540 \\
\hline & Men's alcohol use & -0.034 & 0.094 & 0.7231 & & -0.050 & 0.095 & 0.5989 & \\
\hline & Women's alcohol use & 0.174 & 0.109 & 0.1178 & & 0.203 & 0.108 & 0.0627 & \\
\hline & Men's smoking & 0.058 & 0.063 & 0.3613 & & 0.060 & 0.054 & 0.2663 & \\
\hline & Women's smoking & 0.594 & 0.113 & $<0.0001$ & & 0.455 & 0.094 & $<0.0001$ & \\
\hline \multirow[t]{5}{*}{ Men } & & & & & 0.453 & & & & 0.420 \\
\hline & Men's alcohol use & 0.120 & 0.158 & 0.4502 & & 0.053 & 0.163 & 0.744 & \\
\hline & Women's alcohol use & 0.025 & 0.182 & 0.8894 & & 0.146 & 0.185 & 0.429 & \\
\hline & Men's smoking & 0.209 & 0.105 & 0.0491 & & 0.23 & 0.092 & 0.0122 & \\
\hline & Women's smoking & 0.917 & 0.189 & $<0.0001$ & & 0.575 & 0.161 & 0.0005 & \\
\hline \multirow[t]{5}{*}{ Women } & & & & & 0.542 & & & & 0.581 \\
\hline & Men's alcohol use & -0.134 & 0.073 & 0.0679 & & -0.112 & 0.070 & 0.1089 & \\
\hline & Women's alcohol use & 0.282 & 0.084 & 0.0011 & & 0.244 & 0.079 & 0.0025 & \\
\hline & Men's smoking & -0.060 & 0.048 & 0.2170 & & -0.072 & 0.039 & 0.0686 & \\
\hline & Women's smoking & 0.316 & 0.087 & 0.0004 & & 0.341 & 0.069 & $<0.0001$ & \\
\hline \multicolumn{10}{|c|}{ Adjusted for GDP per capita } \\
\hline \multirow{6}{*}{ Overall } & & & & & 0.612 & & & & 0.587 \\
\hline & Log GDP(US\$) & 2.973 & 0.702 & $<0.0001$ & & 2.464 & 0.685 & 0.0005 & \\
\hline & Men's alcohol use & 0.051 & 0.090 & 0.5726 & & 0.041 & 0.094 & 0.6646 & \\
\hline & Women's alcohol use & -0.007 & 0.110 & 0.9455 & & 0.026 & 0.114 & 0.8218 & \\
\hline & Men's smoking & 0.082 & 0.059 & 0.1649 & & 0.089 & 0.052 & 0.0873 & \\
\hline & Women's smoking & 0.527 & 0.106 & $<0.0001$ & & 0.415 & 0.091 & $<0.0001$ & \\
\hline \multirow[t]{6}{*}{ Men } & & & & & 0.495 & & & & 0.447 \\
\hline & log GDP(US\$) & 3.636 & 1.211 & 0.0033 & & 2.820 & 1.198 & 0.0204 & \\
\hline & Men's alcohol use & 0.230 & 0.156 & 0.1418 & & 0.166 & 0.165 & 0.3165 & \\
\hline & Women's alcohol use & -0.204 & 0.189 & 0.2832 & & -0.069 & 0.199 & 0.7273 & \\
\hline & Men's smoking & 0.244 & 0.101 & 0.0180 & & 0.267 & 0.091 & 0.0039 & \\
\hline & Women's smoking & 0.837 & 0.183 & $<0.0001$ & & 0.533 & 0.158 & 0.0010 & \\
\hline \multirow[t]{6}{*}{ Women } & & & & & 0.606 & & & & 0.634 \\
\hline & Log GDP(US\$) & 2.258 & 0.544 & $<0.0001$ & & 1.968 & 0.495 & 0.0001 & \\
\hline & Men's alcohol use & -0.075 & 0.070 & 0.2841 & & -0.045 & 0.068 & 0.5067 & \\
\hline & Women's alcohol use & 0.153 & 0.085 & 0.0735 & & 0.111 & 0.082 & 0.1791 & \\
\hline & Men's smoking & -0.044 & 0.045 & 0.3316 & & -0.048 & 0.038 & 0.1982 & \\
\hline & Women's smoking & 0.265 & 0.082 & 0.0017 & & 0.305 & 0.065 & $<0.0001$ & \\
\hline
\end{tabular}

\footnotetext{
* In both analyses, alcohol use in 2010 was used.
} 
It is interesting to note that the women's smoking prevalence was consistently associated with the incidence of lung cancer after adjusting for GDP per capita, which was highly correlated with the human development index (Spearman's correlation coefficient $r>0.95$; Pearson's $r>0.90$ ) based on the data in the year 2000 and 2010 (Table 3). The GDP per capita was also significantly associated with the incidence of lung cancer overall, in men and women, and the association was stronger in 2010 than 2000. After adjusting for GDP, the women's alcohol use was no longer significantly associated with the lung cancer incidence in women. In addition, with the data in 2000, the women's smoking had a stronger association with the incidence of lung cancer in women in both models with or without adjusting for the GDP per capita.

\section{The Ratio of Smoking in Men to Women Explained the Gender Difference in Lung Cancer}

The gender difference in the rate of incidence of lung cancer can be partially explained by the difference in smoking rates between men and women (Table 4). Based on the smoking rate and alcohol use in 2010, we found that the ratio of smoking prevalence between men and women was significantly associated with the ratio of the lung cancer incidence between

Table 4. Gender difference in lung cancer incidence and the ratios in smoking and alcohol use between men and women.

\begin{tabular}{|c|c|c|c|c|c|}
\hline Year & Independent variable & Beta & SE & $\boldsymbol{P}$ & $R^{2}$ \\
\hline \multicolumn{6}{|l|}{2010} \\
\hline & The ratio of smoking in men to women * & 0.373 & 0.152 & 0.0155 & 0.0526 \\
\hline & The ratio of alcohol use in men to women & 0.103 & 0.106 & 0.3322 & 0.0086 \\
\hline & The ratio of smoking in men to women * & 0.375 & 0.167 & 0.0272 & 0.0526 \\
\hline & The ratio of alcohol use in men to women & -0.003 & 0.115 & 0.9826 & \\
\hline & GDP per capita $(\$ 1000)$ & 0.002 & 0.145 & 0.9874 & 0.0533 \\
\hline & The ratio of smoking in men to women * & 0.373 & 0.188 & 0.0492 & \\
\hline & The ratio of alcohol use in men to women & 0.000 & 0.115 & 0.9966 & \\
\hline \multicolumn{6}{|l|}{2000} \\
\hline & The ratio of smoking in men to women * & 0.558 & 0.159 & 0.0006 & 0.0970 \\
\hline & The ratio of alcohol use in men to women & 0.104 & 0.105 & 0.3228 & 0.0085 \\
\hline & The ratio of smoking in men to women * & 0.563 & 0.168 & 0.0011 & 0.0971 \\
\hline & The ratio of alcohol use in men to women & -0.009 & 0.106 & 0.9324 & \\
\hline & GDP per capita $(\$ 1000)$ & -0.048 & 0.133 & 0.7168 & 0.1011 \\
\hline & The ratio of smoking in men to women * & 0.535 & 0.194 & 0.0069 & \\
\hline & The ratio of alcohol use in men to women & -0.016 & 0.110 & 0.8853 & \\
\hline
\end{tabular}

* log-transformed; the ratio of lung cancer incidence between men and women was based on the estimates in 2018. 
men and women ( $t=2.46 ; P=0.0155)$. This association was consistent even when adjusting for the gender ratio in alcohol use and the gender ratio in smoking explained about $5.26 \%$ of the variation in the ratio of lung cancer between men and women. We further found that the gender ratio of smoking in 2000 showed a stronger association with the ratio of lung cancer $\left(P=0.0011 ; R^{2}=0.097\right)$ after adjusting for the ratio of alcohol use. Additionally, the gender ratio of smoking explained the $9.7 \%$ of the variation in the ratio of lung cancer incidence. These associations are interesting because cigarette smoking and lung cancer have a certain number of years of latency [12]. However, we did not find a significant association of GDP with the gender ratio in lung cancer even though it was associated with lung cancer in women (Table 4). We noted that GDP was not associated with the gender ratio of lung cancer, although it is significantly associated with the incidence of lung cancer.

\section{DISCUSSION}

We report an analysis of smoking and alcohol use with the incidence of lung cancer at country level and found that the women's smoking rate was associated with the incidence of lung cancer overall, in men and women. The association was stronger when smoking data from 2000 instead of 2010 was used, in particular when adjusting for the GDP per capita, which was also associated with the incidence of lung cancer. We found that the imbalanced incidence between men and women, and the gender ratio of smoking rates could explain about $10 \%$ of the variation in the gender ratio of lung cancer incidence, but GDP per capita was not associated.

That women's smoking rate was associated with the overall, and the observed gender-specific lung cancer incidence is intriguing. While ecological findings may not apply to explain the association of individual behaviors with the risk of lung cancer, it helps provide insight into what we are unable to observe at an individual level, in particular for lung cancer, which is likely, affected by ecological factors such air pollution. Such evidence may help design future studies that can consider the ecological factors. It has been shown that female smokers are 2-fold more likely than men smokers to develop lung cancer especially the small cell carcinoma and squamous cell carcinoma [13], although there are some contradictory reports [14]. However, molecular epidemiological study of a large sample of lung adenocarcinomas showed that female patients tend to have a high frequency of smoking-related KRAS-mutant (G12C) even though they smoked less, suggesting that the female patients of lung cancer are more susceptible to having a smoking-related mutation [15]. While the mechanism underlying the gender difference in lung cancer incidence is not clear, a study has indicated that women have a lower capacity for DNA repair than men [16]. In addition, women's smoking rate was associated with lung cancer incidence even when adjusting for the level of socioeconomic development, which alone has been associated 
with the incidence of lung cancer at both country [17] and individual level even after adjusting for smoking in Europe and Canada [18] and African American [19]. We have to note that this finding at aggregated level cannot be used to explain the association of smoking and risk of lung cancer at individual levels, but it would provide evidence that a study of individual smoking and lung cancer should consider such kind factors at an aggregated level.

In addition, the association of women's smoking with the incidence of lung cancer may have broad implication for translational research into population health. Because women are more at risk for lung cancer, when they are exposed to smoking at an individual level and the prevalence of smoking is rising in the younger women across countries [20]. There is a clear public health need to develop interventions that deter uptake of smoking by young women and for programs to help young women stop smoking.

The findings that the ratio of smoking prevalence in the year 2000 between men and women could explain about $10 \%$ of the variation in the ratio of incidence for lung cancer between men and women is of interest and provide additional evidence that smoking causes lung cancer. With the data from the year 2000, we observed a stronger association of women's smoking with lung cancer incidence, which is probably because the incidence had 18 years lag time, closer to the period of latency for lung cancers.

\section{CONCLUSIONS}

In summary, we provide evidence at an aggregated level that women's smoking prevalence is associated with the incidence of lung cancer; and the association of the gender difference in smoking prevalence with the ratio of lung cancer incidence between men and women provides strong evidence for the relationship between smoking and the risk of lung cancer.

\section{AUTHOR CONTRIBUTIONS}

F.Z. conceived this study; F.Z. and C.Z. acquired the data and performed the analysis; F.Z. and Y.J. interpreted the results; all authors contributed to manuscript writing and critical comments.

\section{CONFLICTS OF INTEREST}

The authors declare that there is no conflict of interest regarding the publication of this paper.

\section{REFERENCES}

1. Institute of Medicine (US) Committee on Health and Behavior: Research, Practice, and Policy. Health and Behavior: The Interplay of Biological, 
Behavioral, and Societal Influences. Washington (DC, US): National Academies Press; 2001.

2. Von Ah D, Ebert S, Ngamvitroj A, Park N, Kang DH. Factors related to cigarette smoking initiation and use among college students. Tob Induc Dis. 2005;3(1):27-40.

3. Substance Abuse and Mental Health Services Administra-tion. Key substance use and mental health indicators in the United States: Results from the 2017 National Survey on Drug Use and Health. Rockville, MD (US): Center for Behavioral Health Statistics and Quality, Substance Abuse and Mental Health Services Administration; 2018. HHS Publication No. SMA 18-5068, NSDUH Series $\mathrm{H}-53$.

4. Islami F, Torre LA, Jemal A. Global trends of lung cancer mortality and smoking prevalence. Transl Lung Cancer Res. 2015;4(4):327-38.

5. Zhang F. Ethnicity, Geographic Location, and Cancer, in Translational Toxicology and Therapeutics: Windows of Developmental Susceptibility in Reproduction and Cancer. Waters M, Hughes CL, editors. New York (US): Wiley; 2017.

6. Doll R, Hill AB. Smoking and carcinoma of the lung; preliminary report. $\mathrm{Br}$ Med J. 1950;2(4682):739-48.

7. Wynder EL, Graham EA. Tobacco smoking as a possible eti-ologic factor in bronchogenic carcinoma; a study of 684 proved cases. J Am Med Assoc. 1950;143(4):329-36.

8. Walser T, Cui X, Yanagawa J, Lee JM, Heinrich E, Lee G, et al. Smoking and lung cancer: the role of inflammation. Proc Am Thorac Soc. 2008;5(8):811-5.

9. Bandera EV, Freudenheim JL, Vena JE. Alcohol consumtion and lung cancer: a review of the epidemiologic evidence. Cancer Epidemiol Biomarkers Prev. 2001;10(8):813-21.

10. Yu SY, Chu YJ, Li WG. Selenium chemo-prevention of liver cancer in animals and possible human applications. Biol Trace Elem Res. 1988;15:231-41.

11 WHO. World health statistics 2018: monitoring health for the SDGs, sustainable development goals. Geneva (Switzerland): World Health Organization; 2018.

12. Weiss W. Cigarette smoking and lung cancer trends. A light at the end of the tunnel? Chest. 1997;111(5):1414-6.

13. Papadopoulos A, Guida F, Leffondré K, Cénée S, Cyr D, Schmaus A. Heavy smoking and lung cancer: are women at higher risk? Result of the ICARE study. Br J Cancer. 2014;110(5):1385-91.

14. De Matteis S, Consonni D, Pesatori AC, Bergen AW, Bertazzi PA, Caporaso NE, et al. Are women who smoke at higher risk for lung cancer than men who smoke? Am J Epidemiol. 2013;177(7):601-12.

15. Dogan S, Shen R, Ang DC, Johnson ML, D'Angelo SP, Paik PK. Molecular epidemiology of EGFR and KRAS mutations in 3,026 lung adeno-carcinomas: higher susceptibility of women to smoking-related KRAS-mutant cancers. Clin Cancer Res. 2012;18(22):6169-77. 
16. Wei Q, Cheng L, Amos CI, Wang LE, Guo Z, Hong WK, et al. Repair of tobacco carcinogen-induced DNA adducts and lung cancer risk: a molecular epidemiologic study. J Natl Cancer Inst. 2000;92(21):1764-72.

17. Wong MCS, Lao XQ, Ho KF, Goggins WB, Tse SLA. Incidence and mortality of lung cancer: global trends and association with socioeconomic status. Sci Rep. 2017;7(1):14300.

18. Hovanec J, Siemiatycki J, Conway DI, Olsson A, Stücker I, Guida F, et al. Lung cancer and socioeconomic status in a pooled analysis of case-control studies. PLoS One. 2018;13(2):e0192999.

19. Aldrich MC, Selvin S, Wrensch MR, Sison JD, Hansen HM, Quesenberry CP Jr, et al. Socioeconomic status and lung cancer: unraveling the contribution of genetic admixture. Am J Public Health. 2013;103(10):e73-80.

20. Roy P, Goel S. Is female smoking rising or declining in India? South Asian J Cancer. 2015;4(2):99-100.

How to cite this article:

Jiang Y, Zhang C, Zhang F. Association of Smoking Prevalence and Alcohol Use with Risk of Lung Cancer: Ecological Evidence. Med One. 2019;4:e190004. https://doi.org/10.20900/mo.20190004 